\title{
Expanded View:
}

\section{Glucomannan preparation}

Dendrobium officinale stem was ground into powder by superfine grinding. Glucomannan from Dendrobium officinale was extracted and purified using the previous method [1].

Fresh tuber of herbaceous perennial konjac was cut into slices and dried in the sun, and then the dried tube was ground into powder by superfine grinding. Dry powder was suspended in $100 \%$ ether (weight/volume; $1: 5)$ and $45 \%$ ethanol $(\mathrm{g} / \mathrm{mL})$ with constant stirring for $24 \mathrm{~h}$ and $1.5 \mathrm{~h}$ respectively. The residue was mixed with 10 times of distilled water $(\mathrm{g} / \mathrm{mL})$ with continuously stirring for $2 \mathrm{~h}$ followed by centrifugation at 4, $800 \mathrm{rpm} / \mathrm{min}$ and collect the supernatant, repeat the extraction for 3 times and combined the supernatants. The supernatant was concentrated by using a rotary evaporator, and then 4 times of $100 \%$ ethanol was slowly added to the concentrate with constant stirring to form a suspension. The suspension was stored at $4{ }^{\circ} \mathrm{C}$ for $24 \mathrm{~h}$, followed by centrifugation at $4,800 \mathrm{rpm} / \mathrm{min}$ for $10 \mathrm{~min}$. The residue was freeze-dried to obtain crude polysaccharides and the purified soluble konjac glucomannan (KGM) was purified according to the previous method [2].

The Aloe vera gel was separated from the whole leaves and homogenized. The homogenate obtained was concentrated to a volume using a rotary evaporator and 5 times of $95 \%(\mathrm{v} / \mathrm{v})$ ethanol was slowly added to the concentrate with continuous stirring to form a suspension. The suspension was stored at $4{ }^{\circ} \mathrm{C}$ for $24 \mathrm{~h}$, followed by 
centrifugation at 4,800 $\mathrm{rpm} / \mathrm{min}$ for $10 \mathrm{~min}$. The residue after the final centrifugation was collected and re-dissolved in deionized water. The newly formed solution was freeze-dried to obtain aloe vera gel soluble glucomannan (AGP) [3].

\section{UPLC-triple-TOF/MS analysis conditions}

\subsection{UPLC-triple-TOF/MS analysis conditions for serum metabolism}

For metabolism analysis, the LC separation was performed with the Shimadzu Nexera X2 UHPLC system (Kyoto, Japan). Chromatographic separation was carried out at $40{ }^{\circ} \mathrm{C}$ with a waters HSS T3 C18 column $(2.1 \mathrm{~mm} \times 100 \mathrm{~mm}, 2 \mu \mathrm{m})$. A gradient program was used at a flow rate of $0.30 \mathrm{~mL} / \mathrm{min}$ and the injection volume was $5 \mu \mathrm{L}$. Mobile phase A was $\mathrm{H}_{2} \mathrm{O}$ mixed with $0.1 \%$ formic acid and mobile phase B was ACN with $0.1 \%$ formic acid for the positive model and $10 \mathrm{mM}$ ammonium acetate water solution and ACN were mobile phases A and B respectively for the negative model. The gradient program was applied as follows: 95\% A/5\% B maintained 2 min, then, increased to $20 \% \mathrm{~A} / 80 \% \mathrm{~B}$ from 2 to $5 \mathrm{~min}$; increased to $10 \% \mathrm{~A} / 90 \% \mathrm{~B}$ from 5 to 12 min; maintained at $10 \% \mathrm{~A} / 90 \% \mathrm{~B}$ from 12 to 14 min; increased to $0 \% \mathrm{~A} / 100 \% \mathrm{~B}$ from 14 to $16 \mathrm{~min}$; maintained at $0 \% \mathrm{~A} / 100 \% \mathrm{~B}$ from 16 to $18 \mathrm{~min}$; decreased to $95 \%$ A/5\% B from 18 to $19 \mathrm{~min}$; maintained at $95 \%$ A/5\% B from 19 to $20 \mathrm{~min}$. For the acquisition of the MS/MS data, the MS system was operated using an AB SCIEX TripleTOF $5600^{+}$mass analyzer with information dependent acquisition (IDA) for ions in both positive and negative modes. Any TOF-MS survey scan peak exceeding $500 \mathrm{cps}$ was selected for the dependent scan in IDA and eight candidate ions were 
allowed per cycle. For positive mode, the collision energy (CE) and collision energy spread (CES) were set to $40 \mathrm{~V}$ and $20 \mathrm{~V}$, respectively. Declustering potential was set to $80 \mathrm{~V}$, and ion spray voltage was set to $+5500 \mathrm{~V}$ with the mass range from 50 to $1000 \mathrm{~m} / \mathrm{z}$. For negative mode, the CE and CES were set to $-40 \mathrm{~V}$ and $20 \mathrm{~V}$, respectively. Declustering potential was set to $-80 \mathrm{~V}$, and ion spray voltage was set to $-4500 \mathrm{~V}$ with a mass range from 50 to $1000 \mathrm{~m} / \mathrm{z}$. The other source parameters were as follows: the ion source gas 1 and gas 2 were set at 50 psi, the curtain gas was set at 35 psi, and the drying temperature was set at $500{ }^{\circ} \mathrm{C}$. Calibration solutions purchased from AB SCIEX were used for instrument calibration, and the high resolution was choosing in the $\mathrm{ESI}^{+}$and $\mathrm{ESI}^{-}$mode.

\subsection{UPLC-triple-TOF/MS analysis conditions for liver metabolism}

For metabolism analysis, the LC separation was performed with the Shimadzu Nexera X2 UHPLC system (Kyoto, Japan). Chromatographic separation was carried out at $40{ }^{\circ} \mathrm{C}$ with a waters HSS T3 $\mathrm{C} 18$ column $(2.1 \mathrm{~mm} \times 100 \mathrm{~mm}, 2 \mu \mathrm{m})$. A gradient program was used at a flow rate of $0.25 \mathrm{~mL} / \mathrm{min}$ and the injection volume was $5 \mu \mathrm{L}$. Mobile phase A was $\mathrm{H}_{2} \mathrm{O}$ mixed with $0.1 \%$ formic acid and mobile phase B was ACN with $0.1 \%$ formic acid for the positive model and $10 \mathrm{mM}$ ammonium acetate water solution and ACN were mobile phases A and B respectively for the negative model. The gradient program was applied as follows: 95\% A/5\% B maintained 2 min, then, increased to $20 \% \mathrm{~A} / 80 \% \mathrm{~B}$ from 2 to $5 \mathrm{~min}$; increased to $10 \% \mathrm{~A} / 90 \% \mathrm{~B}$ from 5 to 12 min; maintained at $10 \% \mathrm{~A} / 90 \% \mathrm{~B}$ from 12 to $14 \mathrm{~min}$; increased to $0 \% \mathrm{~A} / 100 \% \mathrm{~B}$ from 14 to $16 \mathrm{~min}$; maintained at $0 \% \mathrm{~A} / 100 \% \mathrm{~B}$ from 16 to $18 \mathrm{~min}$; decreased to $95 \%$ 
A/5\% B from 18 to 19 min; maintained at $95 \%$ A/5\% B from 19 to $20 \mathrm{~min}$. For the acquisition of the MS/MS data, the MS system was operated using an AB SCIEX TripleTOF $5600^{+}$mass analyzer with information dependent acquisition (IDA) for ions in both positive and negative modes. Any TOF-MS survey scan peak exceeding 500 cps was selected for the dependent scan in IDA and eight candidate ions were allowed per cycle. For positive mode, the collision energy (CE) and collision energy spread (CES) were set to $40 \mathrm{~V}$ and $20 \mathrm{~V}$, respectively. Declustering potential was set to $80 \mathrm{~V}$, and ion spray voltage was set to $+5500 \mathrm{~V}$ with the mass range from 50 to $1000 \mathrm{~m} / \mathrm{z}$. For negative mode, the CE and CES were set to $-40 \mathrm{~V}$ and $20 \mathrm{~V}$, respectively. Declustering potential was set to $-80 \mathrm{~V}$, and ion spray voltage was set to $-4500 \mathrm{~V}$ with a mass range from 50 to $1000 \mathrm{~m} / \mathrm{z}$. The other source parameters were as follows: the ion source gas 1 and gas 2 were set at 50 psi, the curtain gas was set at 35 psi, and the drying temperature was set at $500{ }^{\circ} \mathrm{C}$. Calibration solutions purchased from AB SCIEX were used for instrument calibration, and the high resolution was choosing in the $\mathrm{ESI}^{+}$and $\mathrm{ESI}^{-}$mode.

\subsection{UPLC-triple-TOF/MS analysis conditions for colonic contents metabolism}

For metabolism analysis, the LC separation was performed with the Shimadzu Nexera X2 UHPLC system (Kyoto, Japan). Chromatographic separation was carried out at $40{ }^{\circ} \mathrm{C}$ with a waters HSS T3 C18 column $(2.1 \mathrm{~mm} \times 100 \mathrm{~mm}, 2 \mu \mathrm{m})$. A gradient program was used at a flow rate of $0.30 \mathrm{~mL} / \mathrm{min}$ and the injection volume was $6 \mu \mathrm{L}$. Mobile phase A was $\mathrm{H}_{2} \mathrm{O}$ mixed with $0.1 \%$ formic acid and mobile phase B was ACN with $0.1 \%$ formic acid for the positive model and $10 \mathrm{mM}$ ammonium acetate water 
solution and ACN were mobile phases A and B respectively for the negative model. The gradient program was applied as follows: 95\% A/5\% B maintained 1.5 min, then, increased to $75 \% \mathrm{~A} / 25 \% \mathrm{~B}$ from 1.5 to $2 \mathrm{~min}$; increased to $50 \% \mathrm{~A} / 50 \% \mathrm{~B}$ from 2 to 4 min; increased to $30 \% \mathrm{~A} / 70 \% \mathrm{~B}$ from 4 to $6 \mathrm{~min}$; increased to $20 \% \mathrm{~A} / 80 \%$ B from 6 to 9 min; increased to $5 \% \mathrm{~A} / 95 \% \mathrm{~B}$ from 9 to $13.5 \mathrm{~min}$; maintained at $5 \% \mathrm{~A} / 95 \% \mathrm{~B}$ from 13.5 to $15.5 \mathrm{~min}$; decreased to $95 \% \mathrm{~A} / 5 \% \mathrm{~B}$ from 15.5 to $16 \mathrm{~min}$; maintained at 95\% A/5\% B from 16 to $17 \mathrm{~min}$. For the acquisition of the MS/MS data, the MS system was operated using an AB SCIEX TripleTOF $5600^{+}$mass analyzer with information dependent acquisition (IDA) for ions in both positive and negative modes. Any TOF-MS survey scan peak exceeding $500 \mathrm{cps}$ was selected for the dependent scan in IDA and eight candidate ions were allowed per cycle. For positive mode, the collision energy (CE) and collision energy spread (CES) were set to $40 \mathrm{~V}$ and $20 \mathrm{~V}$, respectively. Declustering potential was set to $80 \mathrm{~V}$, and ion spray voltage was set to $+5500 \mathrm{~V}$ with the mass range from 50 to $1000 \mathrm{~m} / \mathrm{z}$. For negative mode, the CE and CES were set to $-40 \mathrm{~V}$ and $20 \mathrm{~V}$, respectively. Declustering potential was set to $-80 \mathrm{~V}$, and ion spray voltage was set to $-4500 \mathrm{~V}$ with a mass range from 50 to $1000 \mathrm{~m} / \mathrm{z}$. The other source parameters were as follows: the ion source gas 1 and gas 2 were set at 50 psi, the curtain gas was set at 35 psi, and the drying temperature was set at $500{ }^{\circ} \mathrm{C}$. Calibration solutions purchased from AB SCIEX were used for instrument calibration, and the high resolution was choosing in the $\mathrm{ESI}^{+}$and $\mathrm{ESI}^{-}$mode.

\section{UPLC-Q-Trap analysis conditions}

The liquid chromatographic (LC) separation was performed by Shimadzu Nexera 
X2 UHPLC system (Kyoto, Japan). Chromatographic separation was carried out at $40{ }^{\circ} \mathrm{C}$ with a a Shin-pack GIST C18 column $(2.1 \mathrm{~mm} \times 75 \mathrm{~mm}, 2 \mu \mathrm{m})$. For colonic amino acids quantification, a gradient program was used at a flow rate of 0.15 $\mathrm{mL} / \mathrm{min}$ and the injection volume was $2.0 \mu \mathrm{L}$. Mobile phase A was water/methanol (90:10) contained $0.1 \%$ formic acid, Mobile phase B was methanol/water (50:50) with $0.1 \%$ formic acid. The gradient program was applied as follows: $95 \% \mathrm{~A} / 5 \% \mathrm{~B}$ at 0 min, then increased to $70 \% \mathrm{~A} / 30 \% \mathrm{~B}$ from 0 to $0.5 \mathrm{~min}$; increased to $40 \% \mathrm{~A} / 60 \% \mathrm{~B}$ from 0.5 to $1 \mathrm{~min}$; increased to $5 \% \mathrm{~A} / 95 \% \mathrm{~B}$ from 1 to 4 min; maintained $5 \% \mathrm{~A} / 95 \%$ B from 4 to $6 \mathrm{~min}$, returned to $95 \%$ A/5\% B from 6 to $8 \mathrm{~min}$; maintained at $5 \%$ A/95\% B from 8 to 10 min. For serum and liver amino acid quantification, a gradient program was used at a flow rate of $0.10 \mathrm{~mL} / \mathrm{min}$ and the injection volume was $2.0 \mu \mathrm{L}$. Mobile phase A was water/methanol (90:10) contained 0.1\% formic acid, Mobile phase B was methanol/water (50:50) with $0.1 \%$ formic acid. The gradient program was applied as follows: $95 \% \mathrm{~A} / 5 \% \mathrm{~B}$ at 0 min, then increased to $60 \% \mathrm{~A} / 40 \% \mathrm{~B}$ from 0 to $0.5 \mathrm{~min}$; increased to $58 \% \mathrm{~A} / 42 \% \mathrm{~B}$ from 0.5 to $0.75 \mathrm{~min}$; increased to $55 \%$ $\mathrm{A} / 45 \% \mathrm{~B}$ from 0.75 to $1 \mathrm{~min}$; increased to $53 \% \mathrm{~A} / 47 \% \mathrm{~B}$ from 1 to $2 \mathrm{~min}$; maintained $53 \%$ A/47\% B from 2 to 4 min; increased to $43 \%$ A/57\% B from 4 to $5 \mathrm{~min}$; increased to $40 \% \mathrm{~A} / 60 \% \mathrm{~B}$ from 5 to $5.5 \mathrm{~min}$; increased to $38 \% \mathrm{~A} / 62 \%$ B from 5.5 to $6 \mathrm{~min}$; increased to 5\% A/95\% B from 6 to $7 \mathrm{~min}$; maintained 5\% A/95\% B from 7 to $8 \mathrm{~min}$; returned to $95 \% \mathrm{~A} / 5 \% \mathrm{~B}$ from 8 to $8.5 \mathrm{~min}$; maintained at $5 \% \mathrm{~A} / 95 \% \mathrm{~B}$ from 8.5 to 10 min. For the acquisition of the MS/MS data, the MS system was operated using an AB SCIEX Qtrap 4500 mass analyzer with information dependent acquisition (IDA) for 
ions in positive modes. The ion spray voltage was set at $5500 \mathrm{~V}$ while the source temperature was set at $500{ }^{\circ} \mathrm{C}$. The other source parameters were as follows: the ion source gas 1 and gas 2 were set at 50 psi, the curtain gas was set at 20 psi, collision gas was set at medium.The optimization of MRM method parameters was displayed in Table 1.

Table 1 Method conditions for LC-MS/MS analysis

\begin{tabular}{cccccc}
\hline Amino acid & Q1 (Da) & Q3 (Da) & DP $(\mathrm{V})$ & CE $(\mathrm{V})$ & Dwell (msec) \\
\hline Val & 118.000 & 72.000 & 36.00 & 13.00 & 10 \\
Val & 118.000 & 55.000 & 39.00 & 29.00 & 10 \\
Ile & 132.000 & 86.000 & 40.00 & 15.00 & 10 \\
Ile & 132.000 & 69.000 & 40.00 & 24.00 & 10 \\
Leu & 132.000 & 86.000 & 36.00 & 23.00 & 10 \\
Leu & 132.000 & 69.000 & 36.00 & 23.00 & 10 \\
\hline
\end{tabular}

\section{Micro LC-triple-TOF/MS analysis conditions}

Liquid chromatographic (LC) separation was performed with the ekspertTM MicroLC 415 (AB SCIEX, America). Chromatographic separation was carried out at $40{ }^{\circ} \mathrm{C}$ with a ChromXP C18CL column $(0.3 \mathrm{~mm} \times 150 \mathrm{~mm}, 3 \mu \mathrm{m}) . \mathrm{MS} / \mathrm{MS}^{2}$ data acquisition was operated using an AB SCIEX TripleTOF $5600^{+}$mass analyzer with information dependent acquisition (IDA) for ions in positive modes. A gradient program was used at a flow rate of $5 \mu \mathrm{L} / \mathrm{min}$ and the injection volume was $2.0 \mu \mathrm{L}$. Loading program was used at a flow rate of $10 \mu \mathrm{L} / \mathrm{min}$. Mobile phase A was consisted of water/ACN $(98: 2)$ contained $0.1 \%$ formic acid, Mobile phase B was consisted of water/ACN (2:98) contained $0.1 \%$ formic acid. The gradient program was applied as 
follows: $97 \% \mathrm{~A} / 3 \% \mathrm{~B}$ at $0 \mathrm{~min}$, then increased to $75 \% \mathrm{~A} / 25 \% \mathrm{~B}$ from 0 to $45 \mathrm{~min}$; increased to $65 \% \mathrm{~A} / 35 \% \mathrm{~B}$ from 45 to $55 \mathrm{~min}$; increased to $20 \% \mathrm{~A} / 80 \% \mathrm{~B}$ from 55 to $57 \mathrm{~min}$; maintained at $20 \% \mathrm{~A} / 80 \% \mathrm{~B}$ from 57 to $60 \mathrm{~min}$, returned to $97 \% \mathrm{~A} / 3 \% \mathrm{~B}$ from 60 to $61 \mathrm{~min}$; maintained at 97\% A/3\% B from 61 to 65 min. For the acquisition of the MS/MS data, the MS system was operated using an AB SCIEX TripleTOF 5600 mass analyzer with information dependent acquisition (IDA) for ions in both positive and negative modes. Any TOF-MS survey scan peak exceeding 500 cps was selected for dependent scan in IDA and eight candidate ions were allowed per cycle. For positive mode, the collision energy (CE) and collision energy spread (CES) were set to $35 \mathrm{~V}$ and $15 \mathrm{~V}$, respectively. Declustering potential was set to $80 \mathrm{~V}$, and ion spray voltage was set to $+5500 \mathrm{~V}$ with mass range from 400 to $1250 \mathrm{~m} / \mathrm{z}$. The other source parameters were as follows: the ion source gas 1 and gas 2 were set at 50 psi, the curtain gas was set at $35 \mathrm{psi}$, and the drying temperature was set at $500{ }^{\circ} \mathrm{C}$. Calibration solutions purchased from AB SCIEX were used for instrument calibration, and high resolution was chose in the ESI+ mode.

[1] Xing XH, Cui SW, Nie SP, Phillips GO, Goff HD, Wang Q. Study on Dendrobium officinale O-acetyl-glucomannan (Dendronan®): Part I. Extraction, purification, and partial structural characterization. Bioactive Carbohydrates and Dietary Fibre. 2014; 4(1): 74-83.

[2] Shi, X.-D., Yin, J.-Y., Zhang, L.-J., Huang, X.-J., \& Nie, S.-P. (2019). Studies on O-acetyl-glucomannans from Amorphophallus species: Comparison of physicochemical properties and primary structures. Food Hydrocolloids, 89, 503 - 
511.

[3] Shi, X. D., Yin, J. Y., Huang, X. J., Que, Z. Q., \& Nie, S. P. (2018). Structural and conformational characterization of linear O-acetyl-glucomannan purified from gel of Aloe barbadensis Miller. International Journal of Biological Macromolecules, 120, $2373-2380$. 